\title{
Chronic obstructive pulmonary disease: inhale deeply and start to exercise
}

\author{
Henrik Watz
}

Affiliation: Pulmonary Research Institute at LungenClinic Grosshansdorf, Airway Research Center North (ARCN), German Center for Lung Research (DZL), Grosshansdorf, Germany.

Correspondence: Henrik Watz, Pulmonary Research Institute at LungenClinic Grosshansdorf, Woehrendamm 80, D-22927 Grosshansdorf, Germany. E-mail: h.watzapulmoresearch.de

@ERSpublications

Dual bronchodilation results in better lung deflation but similar exercise capacity compared to LAMA monotherapy http://ow.ly/7zL1309M6LK

Cite this article as: Watz $\mathrm{H}$. Chronic obstructive pulmonary disease: inhale deeply and start to exercise. Eur Respir J 2017; 49: 1700424 [https://doi.org/10.1183/13993003.00424-2017].

Chronic obstructive pulmonary disease (COPD) is defined by partially reversible airflow limitation as a result of either small airway obstruction or loss of elastic recoil due to emphysematous lung destruction, or both [1]. Airflow limitation resulting from small airway obstruction is treated with bronchodilators. Resulting improvements in airflow limitation are in turn quantitatively assessed by measuring the forced expiratory volume in $1 \mathrm{~s}(\mathrm{FEV} 1)$, which is the most widely used spirometric measure in COPD. In fact, most of our knowledge about the magnitude of improvements from bronchodilator therapy comes from this standard measurement of lung function. So we know that the simultaneous inhalation of long-acting muscarinic antagonists and long-acting beta- 2 agonists from one inhaler once or twice daily results in improvements in FEV1 that are superior to the inhalation of the individual components [2-5] and clearly exceed the threshold for clinical importance of $\sim 100 \mathrm{~mL}[6]$.

However, lessons learned from respiratory physiology clearly indicate that it is more important to measure the volumes of air that remain in the lungs of patients with COPD rather than to measure the volume that can be exhaled during a forced breathing manoeuvre [7]. These functional consequences of COPD are called lung hyperinflation and air trapping, i.e. an increase in the functional residual capacity and residual volume and decrease in the inspiratory capacity [7]. Lung hyperinflation has been shown to be closely related to exertional dyspnoea, exercise intolerance, physical inactivity and mortality [7]. Hence, pulmonologists began measuring the improvements in lung hyperinflation by bronchodilator therapy and were able to demonstrate that bronchodilators are also effective lung deflators, as they improve functional residual capacity, residual volume and inspiratory capacity $[8,9]$. Furthermore, these improvements in lung hyperinflation lead to improved exercise tolerance, exertional dyspnoea and physical activity in daily life [8-11].

Given the strong link between lung hyperinflation and resulting exercise intolerance, the question arises whether better lung deflation will physiologically translate into better exercise tolerance. In this regard, the pooled MORACTO studies published in the current issue of the European Respiratory Journal by O'Donnell et al. [12] provide an impressively large dataset to study in detail the effects of fixed dual bronchodilator therapy compared to monobronchodilator therapy on exercise intolerance. Besides the strong statistical power to analyse the primary end-points, this dataset permits the analysis of specific subgroups that might benefit from dual bronchodilation compared to monotherapy. Being asked, as a pulmonologist, whether it would be relevant for our patients to have an increase in inspiratory capacity of $0.254 \mathrm{~L}$, which is $0.100 \mathrm{~L}$ better than the improvements observed by monotherapy, it is tempting to answer "yes". Now we see the results of the MORACTO studies, which on average do not show a statistically

Received: Feb 272017 | Accepted: Feb 282017

Conflict of interest: Disclosures can be found alongside this article at erj.ersjournals.com

Copyright @ERS 2017 
significant additional benefit of maximised lung deflation due to the simultaneous inhalation of the approved doses of tiotropium/olodaterol on exercise endurance time compared to tiotropium monotherapy. However, our clinical impression that better lung deflation is conducive to better exercise tolerance is at least partly confirmed by the MORACTO data, as exercise endurance time is numerically better with the approved doses of tiotropium/olodaterol than with monotherapy. Accordingly, numerically more patients experienced improvement in exercise endurance time, as shown by additional analyses summarised in the supplementary material of the article. So how should we interpret the data? First, we need to remember that the MORACTO studies are placebo-controlled cross-over trials with long wash-out periods without any long-acting bronchodilator therapy. Thus, highly symptomatic patients in need of effective lung deflation are likely to be underrepresented in these studies. Secondly, muscle deconditioning is highly prevalent in COPD [13]. To make use of the improved respiratory breathing physiology, exercise training is needed to further improve the exercise endurance time. This approach is subject to a different study protocol combining pharmacological and nonpharmacological treatments [14].

\section{References}

1 Hogg JC. Pathophysiology of airflow limitation in chronic obstructive pulmonary disease. Lancet 2004; 364: 709-721.

2 Bateman ED, Ferguson GT, Barnes N, et al. Dual bronchodilation with QVA149 versus single bronchodilator therapy: the SHINE study. Eur Respir J 2013; 42: 1484-1494.

3 Buhl R, Maltais F, Abrahams R, et al. Tiotropium and olodaterol fixed-dose combination versus mono-components in COPD (GOLD 2-4). Eur Respir J 2015; 45: 969-979.

4 Decramer M, Anzueto A, Kerwin E, et al. Efficacy and safety of umeclidinium plus vilanterol versus tiotropium, vilanterol, or umeclidinium monotherapies over 24 weeks in patients with chronic obstructive pulmonary disease: results from two multicentre, blinded, randomised controlled trials. Lancet Respir Med 2014; 2: 472-486.

5 Singh D, Jones PW, Bateman ED, et al. Efficacy and safety of aclidinium bromide/formoterol fumarate fixed-dose combinations compared with individual components and placebo in patients with COPD (ACLIFORM-COPD): a multicentre, randomised study. BMC Pulm Med 2014; 14: 178.

6 Cazzola M, MacNee W, Martinez FJ, et al. Outcomes for COPD pharmacological trials: from lung function to biomarkers. Eur Respir J 2008; 31: 416-469.

7 Rossi A, Aisanov Z, Avdeev S, et al. Mechanisms, assessment and therapeutic implications of lung hyperinflation in COPD. Respir Med 2015; 109: 785-802.

8 O'Donnell DE, Fluge T, Gerken F, et al. Effects of tiotropium on lung hyperinflation, dyspnoea and exercise tolerance in COPD. Eur Respir J 2004; 23: 832-840.

9 Beeh KM, Watz H, Puente-Maestu L, et al. Aclidinium improves exercise endurance, dyspnea, lung hyperinflation, and physical activity in patients with COPD: a randomized, placebo-controlled, crossover trial. BMC Pulm Med 2014; 14: 209.

10 Watz H, Krippner F, Kirsten A, et al. Indacaterol improves lung hyperinflation and physical activity in patients with moderate chronic obstructive pulmonary disease-a randomized, multicenter, double-blind, placebo-controlled study. BMC Pulm Med 2014; 14: 158.

11 Watz H, Mailander C, Baier M, et al. Effects of indacaterol/glycopyrronium (QVA149) on lung hyperinflation and physical activity in patients with moderate to severe COPD: a randomised, placebo-controlled, crossover study (The MOVE Study). BMC Pulm Med 2016; 16: 95.

12 O'Donnell DE, Casaburi R, Frith P, et al. Effects of combined tiotropium/olodaterol on inspiratory capacity and exercise endurance in COPD. Eur Respir J 2017; 49: 1601348.

13 Maltais F, Decramer M, Casaburi R, et al. An official American Thoracic Society/European Respiratory Society statement: update on limb muscle dysfunction in chronic obstructive pulmonary disease. Am J Respir Crit Care Med 2014; 189: e15-e62.

14 Troosters T, Bourbeau J, Maltais F, et al. Enhancing exercise tolerance and physical activity in COPD with combined pharmacological and non-pharmacological interventions: PHYSACTO randomised, placebo-controlled study design. BMJ Open 2016; 6: e010106. 